\title{
Avaliação da Eficácia de um Programa de Formação sobre Violência contra Crianças e Adolescentes
}

\section{Evaluation of the Effectiveness of a Training Program on Violence against Children and Adolescents}

\author{
Murilo Sabbag Moretti $i^{I}(\mathbb{D}$ \\ Alex Sandro Gomes Pessoa ${ }^{\text {II }}$ (D)
}

\section{PALAVRAS-CHAVE}

- Violência.

- Criança.

- Adolescente.

- Profissionais de Saúde.

- Programa.

- Efetividade.
${ }^{1}$ Universidade do Oeste Paulista, Presidente Prudente, São Paulo, Brasil.

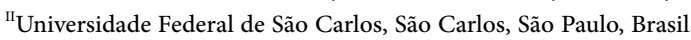

Introdução: O atendimento em serviços de saúde é, muitas vezes, a primeira possibilidade para a identificação de casos de violência contra crianças e adolescentes (VCCA). Sendo assim, o objetivo deste artigo foi avaliar a eficácia de um programa de intervenção desenvolvido para habilitar estudantes e profissionais da área da saúde a reconhecer e encaminhar casos de VCCA. Adicionalmente, buscou-se verificar em qual nível de formação (graduação, pós-graduação ou profissionais em exercício) uma intervenção desse porte teria maior efeito. Método: Trata-se de um estudo quase experimental, delineado a partir de uma análise com um grupo controle (GC) não equivalente. A pesquisa incluiu estudantes de Medicina, pós-graduandos da residência em pediatria e profissionais que atuam em instituições de saúde. Participaram, no total, 105 pessoas, sendo $89 \%$ de mulheres. Os participantes foram posteriormente subdivididos entre o grupo experimental - GE $(n=60)$ e grupo controle - GC $(n=45)$. Um programa de formação, composto por dez sessões (20 horas no total), acerca do tema foi desenvolvido e aplicado a um GE. Para avaliar a efetividade da intervenção, aplicou-se um questionário em períodos previamente determinados (pré-teste e pós-teste). Os dados foram submetidos a análises estatísticas (análises descritivas, teste te de comparações múltiplas de Tukey), por meio do software R. Resultados: O pós-teste revelou alterações estatisticamente significativas em todas as dimensões avaliadas com o GE, o que comprova que a intervenção trouxe mudanças nas concepções prévias que os participantes tinham a respeito da VCCA. Além disso, quando se compararam as respostas obtidas nos questionários entre três grupos do GE (graduandos, pós-graduandos e profissionais em exercício), ficou constatado que não houve diferenças estatísticas intergrupos, o que sugere que programas desse porte têm efeitos positivos independentemente do nível da formação que está sendo ofertada. Conclusões: Este estudo evidenciou que programas de formação podem qualificar a concepção dos estudantes e profissionais da saúde, bem como auxiliar para que se sintam mais preparados para lidar com demandas relativas à VCCA. Cabe, no entanto, um esforço coletivo para que esses conteúdos sejam incorporados de forma propositiva no processo formativo em todos os níveis, da graduação à formação continuada. 


\section{KEYWORDS}

- Violence.

- Children

- Adolescents.

- Health Professionals.

- Program.

- Effectiveness.

\section{ABSTRACT}

Introduction: Assistance in health services is often the first possibility for the identification of cases of Violence Against Children and Teenagers (VACT). Therefore, the aim of this article was to evaluate the effectiveness of an intervention program developed to enable students and health professionals to recognize and report cases of VACT. Additionally, we sought to verify at what level of training (undergraduate, postgraduate or working professional) such intervention would show the greatest effect. Method: This is a quasi-experimental study, of which design was based on the analysis of a non-equivalent Control Group (CG). The research included undergraduate medical students, postgraduate medical students attending pediatric residency and professionals working in health institutions. A total of 105 people participated, of which $89 \%$ were women. The participants were subsequently subdivided between the Experimental Group - EG $(n=60)$ and Control Group - CG $(n=45)$. A training program on the topic, consisting of 10 sessions (20h in total), was developed and applied with an EG. To assess the effectiveness of the intervention, a questionnaire was applied at previously determined periods (pre-test and post-test). The data were submitted to statistical analysis (descriptive analyses, $t$ test and Tukey's multiple comparison test), using the software $R$. Results: The post-test showed statistically significant changes in all dimensions evaluated with the EG, which proves that the intervention resulted in changes regarding the previous conceptions that the participants had about VACT. Additionally, when comparing the responses obtained in the questionnaires between the three EG groups (undergraduate, postgraduate students and working professionals), it was verified that there were no statistical differences between the groups, suggesting that educational programs have positive effects on all levels of training. Conclusions: This study showed that training programs can qualify the conception of students and health professionals, as well as help them feel more prepared to deal with the demands related to VACT. However, a collective effort is needed so that these contents are purposefully incorporated into the training process at all levels, from undergraduate school to continuing education.

Recebido em 12/7/20

Aceito em 14/8/20

\section{INTRODUÇ̃̃O}

A violência é uma das principais causas de morbimortalidade de crianças e adolescentes ${ }^{1}$. Dadas as repercussões na saúde física e psicológica das vítimas, esse é um tema que deve ser amplamente debatido em todos os setores das políticas públicas. Todavia, a formação inicial e continuada dos profissionais de saúde está centrada quase exclusivamente no binômio saúde-doença, o que resulta em despreparo para enfrentar situações como a violência contra crianças e adolescentes (VCCA) ${ }^{2,3}$.

A pesquisa de Koifman et al. ${ }^{4}$, realizada com estudantes do curso de Medicina da Universidade Federal Fluminense (UFF), revelou que a maioria dos participantes (53\%) nunca teve acesso ao tema da VCCA durante a graduação. Somente $9 \%$ mencionaram que o tema foi bem abordado e não apresentaram dúvidas sobre o assunto, e $44 \%$ relataram que não se sentem preparados para lidar com essa questão na prática profissional. Ainda no mesmo estudo, nas entrevistas com médicos residentes, metade relatou não ter tido experiências educativas relativas ao tema, e apenas um participante afirmou ter segurança na identificação dos casos, embora tenha expressado imprecisão em relação à conduta correta a ser seguida caso se deparasse com um caso de $\mathrm{VCCA}^{4}$. Essas constatações são semelhantes aos resultados obtidos na pesquisa conduzida por Margarido et al. ${ }^{5}$ e Nunes et al. ${ }^{6}$ com médicos atuantes no Programa Saúde da Família (atualmente denominado Estratégia Saúde da Família - ESF).

Similarmente, outro estudo realizado com acadêmicos de Medicina, Odontologia e Enfermagem da Universidade Federal de Santa Catarina $(\mathrm{UFSC})^{7}$ evidenciou que o conceito sobre violência entre os entrevistados está relacionado apenas aos danos físicos provocados à vítima. Os sentimentos mais relatados pelos participantes diante de casos de
VCCA foram o medo e a impotência, já que, para os acadêmicos, há um distanciamento do profissional diante dos problemas sociais da população. No currículo desses cursos, concluem os pesquisadores, pouco se discute sobre protocolos de atendimento às vítimas de violência ${ }^{7}$. A falta de informação técnica leva a um despreparo desses profissionais no reconhecimento da VCCA, especialmente nos casos com sinais e sintomas mais sutis ${ }^{4}$. No estudo de Silveira et al. ${ }^{8}$, realizado com profissionais das unidades básicas de saúde (UBS), foi contatado que há uma priorização na atenção aos sinais e sintomas físicos, negligenciando sintomas comportamentais e emocionais.

O levantamento de suspeita depende dos aspectos emocionais dos profissionais e da formação técnica que recebem ${ }^{7,9}$. Além disso, ocorre uma subnotificação dos casos de VCCA pelo medo do envolvimento legal que essa conduta acarreta, bem como pela ausência de mecanismos legais de proteção ao profissional e, até mesmo, pelas ameaças que os profissionais sofrem dos autores da violência ${ }^{10,11}$. A ausência de espaços de formação inicial e continuada, o excesso de atividades na rotina, o tempo limitado para realizar as consultas, a falta de privacidade para estabelecer um diálogo com os pacientes e os recursos reduzidos também colaboram para que haja uma subdetecção dos casos de $\mathrm{VCCA}^{12}$.

Entretanto, o atendimento em pronto-socorro, UBS, ESF, ambulatório ou consultório é, muitas vezes, a primeira possibilidade para o reconhecimento da violência. Os médicos, enfermeiros e outros profissionais de saúde devem estar atentos aos fatores de risco, sinais e sintomas apresentados pelos pacientes. Dessa forma, faz-se necessário incorporar aos currículos de graduação e de pós-graduação formas de amenizar o déficit de aprendizagem teórica e prática no tema da $\mathrm{VCCA}^{4,12,13}$.

REVISTA BRASILEIRA DE EDUCAÇÃO MÉDICA

2 (4) : e127; 2020 
No Brasil, assim como em diversos países do mundo, existem implicações jurídicas para os profissionais da saúde que não notificarem as autoridades públicas mediante um caso suspeito de VCCA. O Estatuto da Criança e do Adolescente (ECA) ${ }^{14}$ no artigo 245 pontua como crime "deixar o médico, professor ou responsável por estabelecimento de atenção à saúde e de ensino fundamental, pré-escola ou creche, de comunicar à autoridade competente os casos de que tenha conhecimento, envolvendo suspeita ou confirmação de maus-tratos contra criança ou adolescente", sob pena de multa de 23 salários de referência, aplicando-se o dobro em caso de reincidência. Portanto, o profissional de saúde precisa lidar com diferentes tipos de sentimentos e situações para os quais não se sente preparado, mas que pode responder judicialmente por seus atos ${ }^{6,9,15}$.

A denúncia de casos de VCCA identificados em serviços de saúde é fundamental para o rompimento da violência e encaminhamento das vítimas à rede de atenção psicossocial. A notificação dos casos, suspeitos ou confirmados, está prevista nos códigos de ética de diversos profissionais da saúde e não configura quebra de sigilo. A Portaria no 104 do Ministério da Saúde prevê a notificação compulsória de casos novos de doenças e agravos, incluindo a violência, e a Portaria $\mathrm{n}^{\circ} 1.968$, de outubro de 2001, estabelece que os responsáveis pelas unidades de saúde do Sistema Único de Saúde (SUS) deverão notificar ao Conselho Tutelar todo caso suspeito ou confirmado de $\operatorname{VCCA}^{9-11}$.

Dadas as possíveis repercussões da violência na vida de crianças e adolescentes, entende-se como imprescindível inserir conteúdos acerca desse fenômeno na formação inicial e continuada de profissionais da saúde. A ausência de espaços de formação repercute na dificuldade do reconhecimento e encaminhamento dos casos, perpetuando a condição de violação de direitos. Desse modo, é imprescindível a elaboração de propostas pedagógicas e modelos educativos sobre o tema que contribuam para a formação desses profissionais, de modo que possam estar mais preparados para quando se depararem com casos de VCCA.

Com base nessas considerações, o objetivo deste artigo foi avaliar a eficácia de um programa de intervenção desenvolvido para habilitar estudantes e profissionais da área da saúde a reconhecer e encaminhar casos de VCCA. Como objetivo secundário, buscou-se verificar em qual nível de formação (graduação, pós-graduação e profissionais em exercício) uma intervenção desse porte teria um efeito maior.

\section{MÉTODO}

Esta pesquisa constitui-se como um estudo quase experimental, delineada a partir de uma análise que empregou pré e pós-testes em um grupo controle não equivalente ${ }^{16}$. Desse modo, a pesquisa de campo foi estruturada com um grupo que participou do programa de intervenção (grupo experimental - GE) e outro que não participou de nenhuma etapa (grupo controle - GC). Ambos os grupos foram avaliados, em termos dos conhecimentos sobre o tema da VCCA, antes e depois do término da intervenção. Embora o trabalho de campo tenha produzido dados qualitativos e quantitativos para avaliação da efetividade do programa, priorizaram-se, neste artigo, os dados provenientes da análise quantitativa.

\section{Participantes e instituições}

A pesquisa incluiu estudantes de Medicina, pós-graduandos da residência médica em pediatria e profissionais que atuam em instituições de saúde. Participaram, no total, 105 pessoas, sendo $89 \%$ de mulheres. Os participantes foram posteriormente subdivididos entre GE ( $\mathrm{n}=60$; Idade $-\mathrm{M}=27,2$; DP $=9,7)$ e GC ( $\mathrm{n}=45$; Idade $\mathrm{M}=28,8 ; \mathrm{DP}=6,03)$.

Como um dos objetivos do estudo foi verificar em qual nível de formação um programa desse porte teria maior efeito, realizouse a intervenção separadamente com os participantes do GE, sendo os subgrupos constituídos da seguinte forma:

- Graduandos: 23 alunos do nono ao décimo segundo termo da Faculdade de Medicina de uma universidade particular do interior de São Paulo fizeram parte do GE e 21 graduandos do oitavo termo de Medicina da mesma universidade compuseram o GC.

- Pós-graduandos: 18 médicos pós-graduandos em Pediatria de um hospital terciário do interior de São Paulo foram alocados no GE e 14 médicos pós-graduandos em Pediatria de uma universidade estadual do interior do Paraná participaram do GC.

- Profissionais de saúde: 19 profissionais médicos, enfermeiros e psicólogos que atuam na rede de atenção municipal de saúde e médicos plantonistas do pronto-socorro pediátrico de um hospital regional de um município de médio porte localizado no interior do estado de São Paulo integraram o GE desse subgrupo e dez profissionais de um hospital terciário do interior de São Paulo compuseram o GC.

Em outras palavras, para cada um dos subgrupos do GE, foi composto um GC com as mesmas características. Como já apresentado, os participantes do GC foram submetidos aos pré e pós-testes, porém não participaram do programa.

\section{Instrumento}

$\mathrm{Na}$ época do início da pesquisa, não foi localizado um instrumento de avaliação sobre concepções de profissionais de saúde acerca do tema da VCCA. Assim, para verificação da eficácia da intervenção, utilizou-se um questionário adaptado de Ferrari et al. ${ }^{17}$. Após as alterações e por se tratar de instrumento que não possui validação no contexto brasileiro, os itens foram submetidos a juízes, ou seja, pesquisadores com notória experiência no campo em que a pesquisa se insere. Tal como preconizado por Hernández-Nieto ${ }^{18}$, os avaliadores analisaram o material com base na clareza de linguagem, pertinência e relevância teórica (um detalhamento pode ser encontrado em Moretti et al. ${ }^{3}$ ).

Em sua versão final, o instrumento foi estruturado em três seções: 1. dados sociodemográficos; 2. concepções sobre VCCA, incluindo as violências física, sexual e psicológica, negligência e abandono; 3 . percepção sobre a atuação do profissional de saúde em casos de VCCA. Os itens das duas últimas seções estavam estruturados no modelo de escala Likert (de cinco pontos), com respostas cujas alternativas variavam entre "discordo totalmente", "discordo", "não concordo nem discordo", "concordo" e "concordo totalmente". Alguns dos itens possuíam valores invertidos, mas as respostas dos participantes indicavam dois aspectos centrais para o estudo: 1. o nível do conhecimento dos profissionais e estudantes acerca da VCCA, incluindo manifestações sintomatológicas, mitos e tabus, tipificação da VCCA, entre outros; 2. conhecimentos relativos à função social dos profissionais de saúde no encaminhamento de denúncias em casos de VCCA, fluxograma de atendimento, dimensões jurídicas e percepção sobre a responsabilidade ética.

REVISTA BRASILEIRA DE EDUCAÇÃo MÉDICA

3 44 (4) : e127; 2020 


\section{Descrição da intervenção}

O programa foi desenvolvido com base em uma revisão não sistematizada da literatura nacional e internacional sobre aspectos teóricos, éticos e procedimentais acerca do trabalho de profissionais da saúde diante de casos de VCCA identificados em contextos institucionais. Além dos conteúdos expositivos, como a apresentação de dados epidemiológicos e diagnósticos clínicos, as sessões privilegiaram atividades interativas e dialógicas entre os participantes. Recorreu-se a estratégias que motivassem a participação e o debate, incluindo o compartilhamento de experiências e desafios com que os participantes já haviam se deparado em situações anteriores. Utilizaram-se recursos imagéticos e sonoros (imagens, vídeos e músicas), slides com conteúdo teórico, estudos de casos clínicos e protocolos recomendados pelo Ministério da Saúde. Complementarmente, ao término de cada sessão, foi fornecida aos participantes uma lista de filmes a respeito do tema abordado naquele encontro.

O programa foi composto por dez sessões, com duração de duas horas cada encontro (totalizando 20 horas), que eram conduzidas uma vez por semana com cada grupo. As sessões foram conduzidas por um médico pediatra com experiência teórica e prática na temática em questão. A síntese da intervenção realizada é descrita no Quadro 1.

\section{Procedimentos e análise dos dados}

Os procedimentos da pesquisa foram realizados em algumas etapas, minuciosamente planejadas pelos pesquisadores. Inicialmente, desenvolveu-se a intervenção, bem como procedeu-se à construção do instrumento que avaliaria os indicadores pré e pós-testes no GE e GC. Antes do início do programa, o GE e o GC responderam, simultaneamente, ao questionário. As sessões do programa ocorreram nos anfiteatros de um hospital ou em auditórios disponibilizados pela Secretaria Municipal de Saúde. Após o encerramento do programa, tanto o GC quanto o GE responderam novamente ao instrumento.

Os dados obtidos foram submetidos à análise estatística descritiva, comparando-se o questionário pré e pós-testes dos grupos participantes. Para verificar se as medidas obtidas antes e depois da implantação do programa foram significativamente diferentes, adotou-se o teste $t$. Esse procedimento é utilizado quando se pretende verificar se a média de uma amostra é igual a um valor de referência ou diferente dele. Quando se identificou diferença entre as médias das categorias, utilizaram-se, complementarmente, testes de comparações múltiplas de Tukey, que permitem identificar essas diferenças entre pares de médias específicos ou em combinações lineares das médias ${ }^{19}$. As análises estatísticas foram realizadas no programa $R$, e considerou-se como estatisticamente significante uma probabilidade inferior ou igual a 0,05 (p-valor $<0,05$ ).

\section{Questões éticas}

$\mathrm{O}$ projeto foi submetido à aprovação do Comitê de Ética em Pesquisa (CEP) com Seres Humanos da Universidade do Oeste Paulista (UNOESTE), que avaliou as questões pertinentes aos preceitos éticos e toda a documentação necessária antes do início de qualquer procedimento de coleta de dados (Certificado de Apresentação para Apreciação Ética: 80281317.2.0000.5515). Os participantes assinaram Termo de Consentimento Livre e Esclarecido (TCLE) expressando concordância com os procedimentos metodológicos. A condução das sessões foi em sala que assegurou as condições de sigilo e adequação ambiental. Após a

\begin{tabular}{|c|c|c|}
\hline \multicolumn{3}{|c|}{$\begin{array}{c}\text { Quadro 1 } \\
\text { Síntese da intervenção }\end{array}$} \\
\hline Sessão & Tema & Objetivo \\
\hline 1 & $\begin{array}{l}\text { A construção social } \\
\text { da infância e da } \\
\text { adolescência }\end{array}$ & $\begin{array}{l}\text { Conceituar infância e adolescência a partir } \\
\text { de uma perspectiva histórica e social. }\end{array}$ \\
\hline 2 & $\begin{array}{l}\text { Os tipos de violência } \\
\text { e suas diversas formas } \\
\text { de manifestação }\end{array}$ & $\begin{array}{l}\text { Reconhecer manifestações da violência } \\
\text { presentes na sociedade e seus impactos } \\
\text { sociais e psicológicos em diferentes } \\
\text { grupos e segmentos sociais. }\end{array}$ \\
\hline 3 & $\begin{array}{l}\text { A violência contra } \\
\text { crianças e adolescentes }\end{array}$ & $\begin{array}{l}\text { Conceituar a violência contra a criança e } \\
\text { o adolescente a partir de suas diferentes } \\
\text { formas de manifestação. }\end{array}$ \\
\hline 4 & Violência física & $\begin{array}{l}\text { Reconhecer lesões, sinais e sintomas } \\
\text { sugestivos de violência física. }\end{array}$ \\
\hline 5 & Violência psicológica & $\begin{array}{l}\text { Reconhecer sintomas, sobretudo } \\
\text { psicológicos, de crianças e adolescentes } \\
\text { expostos à violência psicológica. }\end{array}$ \\
\hline 6 & $\begin{array}{l}\text { Negligência e } \\
\text { abandono }\end{array}$ & $\begin{array}{l}\text { Definir negligência e abandono, bem } \\
\text { como diferenciar situações decorrentes da } \\
\text { precariedade socioeconômica da família. }\end{array}$ \\
\hline 7 & $\begin{array}{l}\text { Violência sexual: } \\
\text { abuso }\end{array}$ & $\begin{array}{l}\text { Classificar e reconhecer a violência sexual } \\
\text { contra a criança e o adolescente levando em } \\
\text { consideração as situações de abuso sexual. }\end{array}$ \\
\hline 8 & $\begin{array}{l}\text { Violência sexual: } \\
\text { exploração }\end{array}$ & $\begin{array}{l}\text { Classificar e reconhecer a violência } \\
\text { sexual contra a criança e o adolescente } \\
\text { levando em consideração as situações de } \\
\text { exploração sexual. }\end{array}$ \\
\hline 9 & $\begin{array}{l}\text { Denúncias e } \\
\text { encaminhamentos }\end{array}$ & $\begin{array}{l}\text { Conhecer as políticas públicas, as } \\
\text { instituições e os equipamentos de proteção } \\
\text { para esses segmentos e o fluxograma de } \\
\text { encaminhamentos de denúncias. }\end{array}$ \\
\hline 10 & $\begin{array}{l}\text { Condutas } \\
\text { autoprotetivas }\end{array}$ & $\begin{array}{l}\text { Compreender as estratégias de condutas } \\
\text { autoprotetivas ante a violência e } \\
\text { aprender como ensiná-las para crianças e } \\
\text { adolescentes. }\end{array}$ \\
\hline
\end{tabular}

Fonte: Elaborado pelos autores.

coleta de dados, foi oportunizado ao GC um curso de formação a respeito do papel dos profissionais da saúde para o encaminhamento de casos de suspeita de VCCA.

\section{RESULTADOS}

Após a aplicação dos questionários nas etapas de pré e pós-testes do GE e GC, foram analisados os itens do instrumento para avaliar a eficácia da intervenção. A Tabela 1 aponta os resultados por categorias nas quais os itens do questionário estavam agrupados: aceitação e naturalização da VCCA, violência física, violência sexual, violência psicológica, negligência e atuação profissional.

Observa-se que no GC não houve diferença estatística entre o pré e o pós-teste em nenhuma das categorias. Já no GE, em todas as dimensões avaliadas houve alterações estatisticamente significativas, o que comprova que a intervenção trouxe mudanças nas concepções prévias que os participantes tinham a respeito do tema da VCCA. Destaca-se a diferença

REVISTA BRASILEIRA DE EDUCAÇÃO MÉDICA

4 44 (4) : e127; 2020 
importante que houve na categoria atuação profissional, que evidencia que, após a formação ofertada, os participantes se sentiram mais aptos e confiantes na atuação de casos de VCCA, bem como mais seguros para o encaminhamento para a rede de proteção.

Quando se compararam as respostas obtidas nos questionários entre os três grupos (graduandos, pós-graduandos e profissionais de saúde) para verificar em qual grupo uma intervenção dessa natureza seria mais eficaz, ficou constatado que não houve diferença estatística intergrupos (Tabela 2), muito embora seja importante destacar que em todos os grupos houve melhoras estaticamente significativas nas concepções sobre VCCA dos participantes, conforme apresentado na Tabela 1. Esses dados evidenciam que modelos educativos ou programas de formação acerca do tema da VCCA podem ser propostos em qualquer fase da formação dos profissionais da saúde (inicial, na pós-graduação e como ações de formação continuada).

\section{DISCUSSÃO}

O Ministério da Saúde define como VCCA "quaisquer atos ou omissões dos pais, parentes, responsáveis, instituições e, em última instância, da sociedade em geral, que redundam em dano físico, emocional, sexual e moral às vítimas"20 É preciso que os profissionais assumam que essa é uma problemática também da área da saúde e entendam que possuem um papel fundamental no que diz respeito ao enfrentamento da VCCA. Em especial, médicos pediatras e enfermeiros, muitas vezes responsáveis pelo primeiro atendimento às vítimas, devem estar atentos a casos que levantarem suspeita ${ }^{4,13}$.

Além de todas as condutas clínicas, é dever do pediatra, em parceria com a equipe, avaliar a gravidade e o risco de revitimização, proceder à denúncia no Conselho Tutelar e comunicar o Sistema de Informação de Agravos de Notificação (Sinan). Tais condutas devem ser tomadas em toda suspeita ou confirmação de violência, além de internação quando há gravidade ou risco para a criança ou o adolescente ${ }^{21,22}$. Entretanto, ainda se observam muitas dificuldades dos profissionais de saúde no tema da VCCA. Por isso, entende-se como imprescindível o aprimoramento dos cursos ofertados nas instituições de ensino responsáveis pela graduação, pós-graduação e educação continuada em saúde. As lacunas no processo formativo levam à falta de informações para o reconhecimento e encaminhamento dos casos ${ }^{23}$.

\begin{tabular}{|c|c|c|c|c|c|c|c|c|c|}
\hline \multicolumn{10}{|c|}{ Avaliação dos pré e pós-testes dos GE e GC } \\
\hline Categoria & Grupos & & $\mathbf{n}$ & Média & Desvio padrão & Erro padrão da média & $\mathbf{t}$ & gl & Sig. (bilateral) \\
\hline \multirow{2}{*}{$\begin{array}{c}\text { Aceitação e } \\
\text { naturalização }\end{array}$} & $\mathrm{GE}^{*}$ & $\begin{array}{l}\text { Pré } \\
\text { Pós }\end{array}$ & 60 & $\begin{array}{l}33,55 \\
37,29\end{array}$ & $\begin{array}{l}4,24 \\
3,64\end{array}$ & $\begin{array}{l}0,55 \\
0,47\end{array}$ & $-5,154$ & 117 & $0,000^{*}$ \\
\hline & GC & $\begin{array}{l}\text { Pré } \\
\text { Pós }\end{array}$ & 45 & $\begin{array}{l}33,13 \\
33,07\end{array}$ & $\begin{array}{l}4,07 \\
3,97\end{array}$ & $\begin{array}{l}0,61 \\
0,59\end{array}$ & 0,079 & 88 & 0,937 \\
\hline \multirow{2}{*}{ Violência física } & $\mathrm{GE}^{*}$ & $\begin{array}{l}\text { Pré } \\
\text { Pós }\end{array}$ & 60 & $\begin{array}{l}13,22 \\
15,58\end{array}$ & $\begin{array}{l}2,89 \\
2,53\end{array}$ & $\begin{array}{l}0,37 \\
0,33\end{array}$ & $-4,777$ & 118 & $0,000^{*}$ \\
\hline & GC & $\begin{array}{l}\text { Pré } \\
\text { Pós }\end{array}$ & 45 & $\begin{array}{l}13,16 \\
13,16\end{array}$ & $\begin{array}{l}2,94 \\
3,25\end{array}$ & $\begin{array}{l}0,44 \\
0,49\end{array}$ & 0,000 & 88 & 1,000 \\
\hline \multirow{2}{*}{ Violência sexual } & $\mathrm{GE}^{*}$ & $\begin{array}{l}\text { Pré } \\
\text { Pós }\end{array}$ & 60 & $\begin{array}{l}31,50 \\
35,38\end{array}$ & $\begin{array}{l}3,07 \\
4,85\end{array}$ & $\begin{array}{l}0,40 \\
0,63\end{array}$ & $-5,241$ & 100 & $0,000^{*}$ \\
\hline & GC & $\begin{array}{l}\text { Pré } \\
\text { Pós }\end{array}$ & 45 & $\begin{array}{l}31,67 \\
31,22\end{array}$ & $\begin{array}{l}3,11 \\
3,46\end{array}$ & $\begin{array}{l}0,46 \\
0,52\end{array}$ & 0,640 & 88 & 0,524 \\
\hline \multirow{2}{*}{$\begin{array}{l}\text { Violência } \\
\text { psicológica }\end{array}$} & $\mathrm{GE}^{*}$ & $\begin{array}{l}\text { Pré } \\
\text { Pós }\end{array}$ & 60 & $\begin{array}{l}14,28 \\
16,45\end{array}$ & $\begin{array}{l}1,82 \\
2,42\end{array}$ & $\begin{array}{l}0,24 \\
0,31\end{array}$ & $-5,542$ & 110 & $0,000^{*}$ \\
\hline & GC & $\begin{array}{l}\text { Pré } \\
\text { Pós }\end{array}$ & 45 & $\begin{array}{l}14,56 \\
14,16\end{array}$ & $\begin{array}{l}1,67 \\
1,98\end{array}$ & $\begin{array}{l}0,25 \\
0,29\end{array}$ & 1,036 & 88 & 0,303 \\
\hline Negligência & $\mathrm{GE}^{*}$ & $\begin{array}{l}\text { Pré } \\
\text { Pós }\end{array}$ & 60 & $\begin{array}{l}17,02 \\
18,17\end{array}$ & $\begin{array}{l}2,07 \\
2,40\end{array}$ & $\begin{array}{l}0,27 \\
0,31\end{array}$ & $-2,809$ & 118 & $0,006^{*}$ \\
\hline (1) & GC & $\begin{array}{l}\text { Pré } \\
\text { Pós }\end{array}$ & 45 & $\begin{array}{l}16,91 \\
16,84\end{array}$ & $\begin{array}{l}2,04 \\
2,20\end{array}$ & $\begin{array}{l}0,30 \\
0,33\end{array}$ & 0,149 & 88 & 0,882 \\
\hline Atuacão profissional & $\mathrm{GE}^{*}$ & $\begin{array}{l}\text { Pré } \\
\text { Pós }\end{array}$ & 60 & $\begin{array}{l}27,13 \\
34,10\end{array}$ & $\begin{array}{l}3,11 \\
3,29\end{array}$ & $\begin{array}{l}0,40 \\
0,42\end{array}$ & $-11,924$ & 118 & $0,000^{*}$ \\
\hline 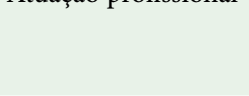 & GC & $\begin{array}{l}\text { Pré } \\
\text { Pós }\end{array}$ & 45 & $\begin{array}{l}28,60 \\
28,84\end{array}$ & $\begin{array}{l}2,77 \\
3,13\end{array}$ & 0,41 & $-0,392$ & 88 & 0,696 \\
\hline
\end{tabular}

${ }^{*} \mathrm{p}<0,05$ (intervalo de confiança de 95\%).

Fonte: Elaborada pelos autores. 


\begin{tabular}{|c|c|c|c|c|c|c|c|c|c|}
\hline \multicolumn{10}{|c|}{$\begin{array}{c}\text { Tabela } 2 \\
\text { Comparação da eficácia da intervenção entre os três grupos participantes }\end{array}$} \\
\hline Grupo & & $\mathbf{N}$ & Média & Desvio padrão & Erro padrão & $\begin{array}{l}\text { Intervalo de } \\
\text { limite inferior }\end{array}$ & $\begin{array}{l}\text { Intervalo de limite } \\
\text { superior }\end{array}$ & Mínimo & Máximo \\
\hline \multirow[b]{2}{*}{ Graduandos } & Pré & 44 & 3,46 & 0,20 & 0,03 & 3,40 & 3,52 & 3,12 & 3,89 \\
\hline & Pós & & 3,75 & 0,42 & 0,06 & 3,62 & 3,88 & 2,97 & 4,57 \\
\hline \multirow[b]{2}{*}{ Pós-graduandos } & Pré & \multirow[b]{2}{*}{32} & 3,54 & 0,27 & 0,05 & 3,44 & 3,63 & 3,02 & 4,07 \\
\hline & Pós & & 3,80 & 0,39 & 0,07 & 3,66 & 3,94 & 3,16 & 4,79 \\
\hline \multirow{2}{*}{$\begin{array}{l}\text { Profissionais em } \\
\text { exercício }\end{array}$} & Pré & \multirow[b]{2}{*}{29} & 3,51 & 0,27 & 0,05 & 3,40 & 3,61 & 2,93 & 3,98 \\
\hline & Pós & & 3,78 & 0,36 & 0,07 & 3,65 & 3,92 & 3,10 & 4,40 \\
\hline
\end{tabular}

${ }^{*} \mathrm{p}<0,05$ (intervalo de confiança de $95 \%$ ).

Fonte: Elaborada pelos autores.

Esta pesquisa revelou que um programa de formação bem estruturado produz mudanças importantes na concepção dos profissionais e estudantes da saúde sobre a VCCA, bem como colabora para que se sintam mais preparados para lidar com essas demandas. Apesar das constatações provenientes desta investigação, Christian ${ }^{24}$ alega que, no contexto internacional, inexistem levantamentos sobre a quantidade e qualidade de programas elaborados especificamente para preparar estudantes de Medicina no tema da VCCA. $\mathrm{Na}$ mesma direção, Gebara e Lourenço ${ }^{25}$ afirmam que os profissionais da saúde do contexto brasileiro não têm espaços formativos para habilitá-los para lidar com esse tipo de demanda, e, em muitos casos, os conhecimentos sobre o tema ocorrem por iniciativa própria.

Estudos da década de 1990, como o de Dubowitz e Black ${ }^{26}$,já apontavam que o oferecimento de uma série de seis seminários, com a duração de 90 minutos cada sessão, já era capaz de ampliar significativamente o conhecimento de estudantes das residências médicas sobre o tema de violação de direitos de criança e adolescentes. Os achados do presente estudo robustecem essa afirmativa, na medida em que um programa de 20 horas se mostrou eficaz e bem-sucedido nessa empreitada. Além disso, os resultados positivos também se estenderam aos estudantes de graduação e profissionais que atuam em diferentes políticas públicas de saúde.

Lise e Motta $^{27}$ afirmam que a VCCA atinge todas as camadas socioeconômicas, étnicas e religiosas. Entretanto, seus efeitos são ainda mais nevrálgicos em grupos expostos a situações de vulnerabilidade social, sobretudo provenientes de famílias que enfrentam desemprego, baixo nível de escolaridade dos cuidadores e escassez de recursos. Sobre esse assunto, Christian ${ }^{24}$ alerta que os estudantes e profissionais da saúde, sobretudo da área da medicina, podem não compreender a complexidade da VCCA justamente por serem provenientes de grupos mais favorecidos socioeconomicamente. Faz-se necessário que os programas de intervenção voltados para ações formativas desse contingente contextualizem, de forma não estigmatizada, a realidade sociocultural das famílias que, por vezes, também se tornam vítimas de um modo de organização social perverso, excludente e promotor de violência.

Uma tarefa inadiável para as instituições de ensino que ofertam cursos de formação inicial e continuada dos profissionais da saúde é a inserção de disciplinas e espaços formativos permanentes que contemplem esses temas ${ }^{28}$. Todavia, para Margarido et al. ${ }^{5}$, os currículos de formação de profissionais da medicina insistem em programas de ensino descontextualizados e acríticos da realidade social. Ainda de acordo com os autores supracitados, os currículos desses cursos respondem, majoritariamente, a demandas do mercado e a práticas elitistas, o que acaba gerando "um hiato entre o contingente de egressos dos centros formadores e as demandas dos serviços de saúde pública" (p. 408).

Este artigo não tem como finalidade defender que é uma responsabilidade exclusiva dos profissionais da saúde atuar na identificação de casos de VCCA existentes. Mas, sem dúvida, esses profissionais podem ser decisivos no reconhecimento dessa realidade e fortalecer, sobremaneira, a rede de proteção das vítimas. Este estudo evidencia que programas de formação podem qualificar a concepção dos profissionais, bem como auxiliar para que se sintam mais preparados para lidar com essas demandas. Cabe, no entanto, um esforço coletivo para que esses conteúdos sejam incorporados de forma propositiva no processo formativo, em todos os níveis, da graduação à formação continuada.

\section{CONSIDERAÇÕES FINAIS}

O objetivo da pesquisa foi avaliar a eficácia um modelo de intervenção, voltado para estudantes e profissionais da área da saúde, capaz de auxiliálos na identificação e no encaminhamento de casos de VCCA. Além disso, buscou-se verificar em qual nível de formação um programa desse porte seria mais eficaz. Ficou demonstrado, por meio das análises estatísticas, que o programa apresentou eficácia e atingiu os objetivos propostos, ou seja, alterou positivamente as concepções prévias dos estudantes e profissionais e os preparou para a condução dos casos e o enfrentamento da VCCA. Também foi constatado que programas desse porte produzem resultados positivos em estudantes e profissionais com diferentes tipos de formação e experiência, o que sugere que ações educativas sobre VCCA sejam inseridas em programas de formação inicial e continuada dos cursos de formação em saúde.

Algumas limitações desta pesquisa podem ser observadas, como a impossibilidade de implementar uma avaliação do nível follow-up ou mesmo acompanhar os profissionais e estudantes longitudinalmente para verificar a aplicação prática dos conhecimentos adquiridos no programa. Recomenda-se, desse modo, que estudos futuros acompanhem e avaliem o desempenho dos estudantes e profissionais em situações reais. Também cabe notar que existem questionamentos acerca dos ganhos obtidos na participação de apenas um programa de curta duração, como é o caso deste

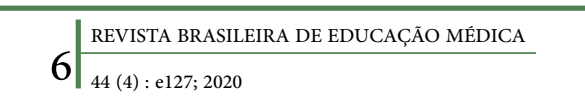


estudo. Há, na literatura especializada, amplo debate sobre a necessidade de que ações desse porte sejam inseridas de forma intermitente na formação dos profissionais da saúde. Portanto, esta investigação lança novas perguntas investigativas e provoca os pesquisadores da área a se debruçar nos dilemas que ainda persistem sobre a temática.

\section{AGRADECIMENTOS}

Agradecemos a Jhully Cristine Ananias Boaro e Amanda Barbosa Ferrador por todo o suporte técnico prestado na finalização do manuscrito e à Coordenação de Aperfeiçoamento de Pessoal de Nível Superior Brasil (Capes) o aporte financeiro - Código de Financiamento 001.

\section{REFERÊNCIAS}

1. Machado JC, Rodrigues VP, Viela ABA, Simões AV, Morais RLGL, Rocha EM. Violência intrafamiliar e as estratégias de atuação da equipe de saúde da família. Saúde Soc. 2014;23(3): 828-40.

2. Malta DC, Mascarenhas MDM, Bernal RTI, Viegas APB, Sá NNB, Silva Junior JB. Acidentes e violência na infância: evidências do inquérito sobre atendimentos de emergência por causas externas. Cienc Saude Colet. 2012;17(9):2247-58.

3. Moretti MS, Medeiros, JK, Pessoa, ASG, Koller, SH. Identificação e encaminhamentos de casos de violência contra crianças no contexto das políticas de saúde. In: Vestena CLB, D’Aroz, MS, Costa-Lobo C, org. As Crianças: Infância, Pobreza e Qualidade de Vida. Curitiba: CRV;2019. p.167-186.

4. Koifman L, Menezes RM, Bohrer KR. Abordagem do tema "violência contra a criança” no curso de Medicina da Universidade Federal Fluminense. Rev Bras Educ Med. 2012;36(2):172-9.

5. Margarido A, Próspero ENS, Grillo LP. Violência doméstica contra crianças e adolescentes: formação e conhecimento dos médicos. Psicol Argum. 2013;31(74):405-14.

6. Nunes CB, Sarti AA, Ohara CVS. Concepções de profissionais de saúde sobre a violência intrafamiliar contra a criança e o adolescente. Rev Lat Am Enfermagem. 2008;16(1):136-41.

7. Rosa R, Boing AF, Schraiber LB, Coelho EBS. Violência: conceito e vivência entre acadêmicos da área da saúde. Interface Comun Saúde Educ. 2010;14(32):81-90 [acesso em 2 out. 2017]. Disponível em: https://www. scielo.br/scielo.php?script=sci_arttext\&pid=S1414-32832010000100007

8. Silveira TB, Oliveira AMN, Algeri S, Susin LRO, Baisch ALM, Marques LA, et al. The invisibility of psychological violence against children. J Hum Growth Dev. 2016;26(3):345-51.

9. Calza TZ, Dell'Aglio DD, Sarriera JC. Direitos da criança e do adolescente e maus-tratos: epidemiologia e notificação. Rev SPAGESP. 2016;17(1):14-27.

10. Cocco M, Silva EB, Jahn AC. Abordagem dos profissionais de saúde em instituições hospitalares a crianças e adolescentes vítimas de violência. Rev Eletrônica Enferm. 2010;12(3):491-7.

11. Garbin CAS, Dias IA, Rovida TAS, Garbin AJI. Desafios do profissional de saúde na notificação da violência: obrigatoriedade, efetivação e encaminhamento. Cienc Saude Colet. 2015;20(6):1879-90.

12. Moura ATMS, Moraes CL, Reichenheim ME. Detecção de maustratos contra a criança: oportunidades perdidas em servições de emergência na cidade do Rio de Janeiro, Brasil. Cad Saude Publica 2008;24(12):2926-36.

13. Paiva C, Zaher VL. Violência contra crianças: o atendimento médico e o atendimento pericial. Saúde, Ética \& Justiça. 2012;17(1):12-20.

14. Brasil. Lei ${ }^{\circ}$ 8.069, de 13 de julho de 1990. Dispõe sobre o Estatuto da Criança e do Adolescente e dá outras providências. Diário Oficial [da] República Federativa do Brasil, Brasília, DF; 16 jul. 1990.

15. Nunes CB, Sarti CA, Ohara CVS. Profissionais de saúde e violência intrafamiliar contra a criança e adolescente. Acta Paul Enferm. 2009;22(ed esp):903-8.

16. Cozby PC. Métodos de pesquisa em ciências do comportamento. São Paulo: Atlas; 2009.

17. Ferrari IMSF, Priolo Filho SR, Brino RF. Questionário sobre violência intrafamiliar: confiabilidade de um instrumento sobre crenças. Psicol Teor Prát. 2016;18(3):54-65.

18. Hernández-Nieto RA. Contributions to statistical analysis. Mérida: Universidade de Los Andes; 2002.

19. Vieira S. Bioestatística: tópicos avançados. Rio de Janeiro: Campus; 2003.

20. Brasil. Linha de cuidado para a atenção integral de crianças, adolescentes e suas famílias em situação de violências. Brasília: Ministério da Saúde; 2010.

21. Pfeiffer L, Rosário NA, Cat MNL. Violência contra crianças e adolescentes: proposta de classificação dos níveis de gravidade. Rev Paul Pediatr. 2011;29(4):477-82.

22. Sociedade Brasileira de Pediatria. Protocolo de abordagem da criança ou adolescente vítima de violência doméstica. Rio de Janeiro: SBP; 2018.

23. Bannwart TH, Brino RF. Dificuldades enfrentadas para identificar e notificar casos de maus-tratos contra crianças e/ou adolescentes sob a óptica de médicos pediatras. Rev Paul Pediatr. 2011;29(2):138-45.

24. Christian CW. Professional education in child abuse and neglect. Pediatrics. 2008;122(1):S13-7.

25. Gebara CFP, Lourenço LM. Crenças de profissionais da saúde sobre violência doméstica contra crianças e adolescentes. Psicol Pesq. 2008;2(1):27-39.

26. Dubowitz H, Black M. Teaching pediatric about child maltreatment. J Dev Behav Pediatr. 1991;12(5):305-7.

27. Lise F, Motta MGC. Violência doméstica infantil: abordagem da enfermagem. Acta Sci. 2012;34(1):53-8.

28. Rocha PCX, Moraes CL. Violência familiar contra a criança e perspectivas de intervenção do Programa de Saúde da Família: a experiência do PMF/Niterói (RJ, Brasil). Cien Saude Colet. 2011;16(7):3286-96.

\section{CONTRIBUIÇÃO DOS AUTORES}

Os autores elaboraram conjuntamente o projeto de pesquisa, o modelo de intervenção, o questionário empregado, as análises dos dados e a preparação do manuscrito. O responsável pela condução das sessões do programa e da coleta de dados foi o primeiro autor.

\section{CONFLITO DE INTERESSES}

Os autores declaram não haver conflito de interesses neste estudo.

\section{ENDEREÇO PARA CORRESPONDÊNCIA}

Murilo Sabbag Moretti. Faculdade de Medicina da Universidade do Oeste Paulista, Rua José Bongiovani, 700, Cidade Universitária, Presidente Prudente, SP, Brasil. CEP: 19050-920.

E-mail: murilomoretti@gmail.com 